\title{
Self-Expansion in Experiential Consumption: An Abstract
}

\author{
Mark J. Pelletier and Joel E. Collier
}

\begin{abstract}
Experiential consumption represents a unique, and exceedingly popular, type of marketing behavior. Existing theory and research in the field of psychology suggest that one of the primary drivers of experiential consumption could be the concept of self-expansion. However, self-expansion as a factor in experiential consumption research has yet to be fully explored. The present research examines the following questions: What type of experiences lead to higher levels of self-expansion? What factors lead to self-expansion and how does self-expansion influence outcome variables such as evangelizing, price consciousness, and repurchase intention? Using a scale adapted from social psychology, self-expansion, as it relates to experiential consumption, is explored, levels of self-expansion stemming from different experiential categories are compared, and the influence of self-expansion on outcome variables is examined.
\end{abstract}

\author{
M.J. Pelletier $(\varangle)$ \\ Radford University, Radford, VA, USA \\ e-mail: mpelletier@radford.edu \\ J.E. Collier \\ Mississippi State University, Starkville, MS, USA \\ e-mail: jcollier@business.msstate.edu
}

